\title{
ASTEROID FAMILIES
}

\author{
VINCENZO ZAPPALÀ AND ALBERTO CELLINO \\ Osservatorio Astronomico di Torino
strada osservatorio 20, I-10025 Pino Torinese (TO), Italy \\ E-mail ZAPPALA@TO.ASTRO.IT
}

\begin{abstract}
In spite of their widely recognized importance in the framework of the modern understanding of the asteroidal population and its collisional evolution, asteroid dynamical families have long been a puzzling subject of research, due to the disagreement among the family lists published by different authors. In the present review, the definition and meaning of asteroid families are critically discussed, as well as the various problems which have to be faced by any suitable technique of family identification. In this respect, major improvements have been achieved during the last few years. The most recent family searches show an excellent agreement both in the number of reliably identified families, and in their members. Moreover, the overall performances of the most recent techniques of family identification have been tested by means of numerical simulations, with encouraging results. For these reasons, we believe that we are presently at the beginning of an era in which detailed physical studies of families can be attempted, and observational campaigns can be planned on the basis of solid evidence, like in the case of the recent spectacular results obtained for the family associated to the large asteroid 4 Vesta.
\end{abstract}

\section{Introduction}

Asteroid dynamical families have received a renewed interest recently, due to the recognition of their intrinsic importance in the framework of modern planetary research. Families are thought to be the outcomes of the catastrophic disruption of a number of sizable parent bodies. For this reason, they provide obvious opportunities for the studies of the mineralogical composition of asteroids, since many family members are made of material coming from the inner layers of their parent body, and put at the same time some essential constraints for any model of the collisional evolution of the asteroid belt. In addition to these well known facts, more recently it has been pointed out that families may be important also for the origin of meteorites, and more generally the interrelationships among minor bodies of the Solar System. As a matter of fact, simple estimates lead to conclude that in some cases the impacts responsible of the formation of a family within the asteroid main belt may also have injected a fraction of fragments into some of the most important resonances, like the $3 / 1$ mean motion resonance with Jupiter at $2.5 \mathrm{AU}$, and the $\nu_{6}$ secular resonance. As a consequence, through dynamical processes which have been recently extensively investigated (see Greenberg and Nolan, 1989, and Farinella et al., in this volume), many fragments may have been injected into the inner regions of the solar system, where they may be discovered as Near-Earth-Asteroids, or even collide with terrestrial planets. An excellent example of these interrelationships among asteroid families, NEA asteroids and meteorites is given by the Vesta family, whose collisional origin has been recently confirmed by observations (Binzel and $\mathrm{Xu}, 1993$; Burbine and Binzel, this volume). As a matter of fact, in the case 
of Vesta we may sketch a general scenario which accounts for all the observational constraints : the existence of the family, originated by an energetic cratering event; the existence of an hemispheric-scale albedo variegation on Vesta (clearly indicated by polarimetric and photometric data, see Cellino et al., 1987, and Broglia and Manara, 1989), which could be related to the presence of the crater; the existence of a definite class of meteorites, the eucrites, and of a number of NEAs (Cruikshank et al., 1991) which both exhibit a basaltic composition, and may plausibly be considered as originating from the fraction of high-speed fragments which were injected into the chaotic zones associated to the $3 / 1$ and $\nu_{6}$ resonances.

These considerations show how the study of asteroid families may be fruitful for our general understanding of many evolutionary processes active in the solar system, allowing to identify large-scale collisions as one of the main mechanisms which have shaped a number of apparently unrelated observational evidences which are often separately studied by different branches of planetary research. The recognition of the unifying role played by collisional evolution is actually one of the main achievements of modern planetary science.

However, the recognition of the importance of asteroid families should lead us to carefully take into account all the reasons which have until recently prevented us from establishing a well defined and coherent identification of those which are presently recognizable within the asteroid belt. In spite of the noticeable number of papers devoted by many authors to the problem of family identification - starting from the pioneering work of Hirayama at the beginning of the present century it has not been possible for a long time to obtain an unambiguous list of families generally accepted by the majority of the scientists active in this field of research.

The present review is aimed at crytically re-analyzing the overall topic of the asteroid family identification and analysis. We start from the different meaning of the definition of families, depending upon the point of view (statistical or physical) adopted for this purpose. We review the problems encountered when an unambiguous identification of families is attempted, and we examine the current state of the art, showing that some important improvements have been obtained during the last few years by means of new methods, devised by different authors. Finally, we discuss what we can learn from a physical analysis of the presently known families, and we examine a list of open questions which still wait for a definitive answer. We can anticipate here that we hope to be able to convince the reader that the general understanding of asteroid families is presently much better than it used to be until a few years ago; in our opinion, we are at the beginning of an era in which the physical information hidden within families is going to be fully exploited. This is a consequence of the improvements in the techniques of identification of families and family memberships. The results start to be supported by some adequate observational evidence, and can be now used for testing the predictions of the presently available physical models of collisional events.

\section{What is a Family?}

It is important to briefly discuss what is the meaning of the word "family". In fact, sometimes this word is used in slightly different ways by the specialists of dynamics, and by the experts of different branches of asteroid physics. From a dynamical 
point of view, families are groups of asteroids having orbital proper elements $a^{\prime}, e^{\prime}$, $i^{\prime}$ (proper semimajor axis, eccentricity and inclination, respectively) very similar. From this point of view, the main feature of families is to be statistically significant, that is clearly separable from random clusterings of objects in the space of proper elements. The immediate interpretation is that they are the outcome of the disruption of single parent bodies, since the laws of dynamics predict that fragments ejected with relatively low velocities, will conserve approximately the proper elements of their parent over long time scales. The general subject of asteroid proper elements is extensively covered by the Knežević and Milani chapter in this volume. For this reason, we do not enter here into details. We only recall that the Gauss equations connect the components of the post-breakup ejection velocities of the fragments from a catastrophic collision with the resulting differences in their proper elements $\delta a^{\prime}, \delta e^{\prime}, \delta i^{\prime}$ (at least in the frame of the linear secular perturbation theory, see Brouwer, 1951; see also Zappalà et al., 1984, and Chapman et al., 1989).

From the point of view of the physics of asteroids, the meaning of families is just related to their collisional origin; in this sense, a set of fragments directly deriving from the disruption of a single parent body constitutes a family.

Even if the dynamical and physical definitions may appear essentially coincident, this is not always true in practice, and some misunderstandings have actually become apparent in the past. As a matter of fact, not always the two definitions of family lead to the same results : on one hand, there may be cases in which statistically significant groupings of objects do not share a common collisional origin; in other cases, "true" collisional families may be not identified on a purely statistical ground. Of course, mainly in the former case, a crucial role is played by the statistical criteria adopted for the identification. Actually, the procedures have to be carefully devised, in order to avoid to accept as significant, some groupings which could be more correctly rejected on the basis of improved criteria. On the other hand, some difficulties are intrinsic, and are a consequence of the actual structure of the asteroid belt. As an example, the Phocaea region (the populated zone located at semimajor axis values of 2.3-2.4 $\mathrm{AU}$, and at high inclination values beyond $20^{\circ}$ ) is largely isolated by the rest of the belt. In such a case, since the only objects present there belong to this "populated island" in the space of proper elements, statistics might lead to accept that they form a family; this result, however, remains questionable, since there are reasons to believe that the Phocaea "island" is actually only a consequence of the depletion of the neighbouring regions due to the occurrence of important secular resonances. On the other hand, at the high inclination values of the Phocaea region, the proper elements used so far are not sufficiently precise to derive any definite conclusion. The situation should improve in the near future, due to the recent availability of a new large set of proper elements of good quality for high inclination objects (Lemaitre and Morbidelli, 1994). A preliminary analysis of these data seems to suggest that at least some objects in the Phocaea "island" may actually belong to a real family.

In order to avoid any misunderstanding due to an improper use of the word "family", Farinella et al. (1992) have proposed a new nomenclature. According to these authors, the term "family" itself should be used only in the cases in which the statistical and physical definitions are coincident. This means that "families" 


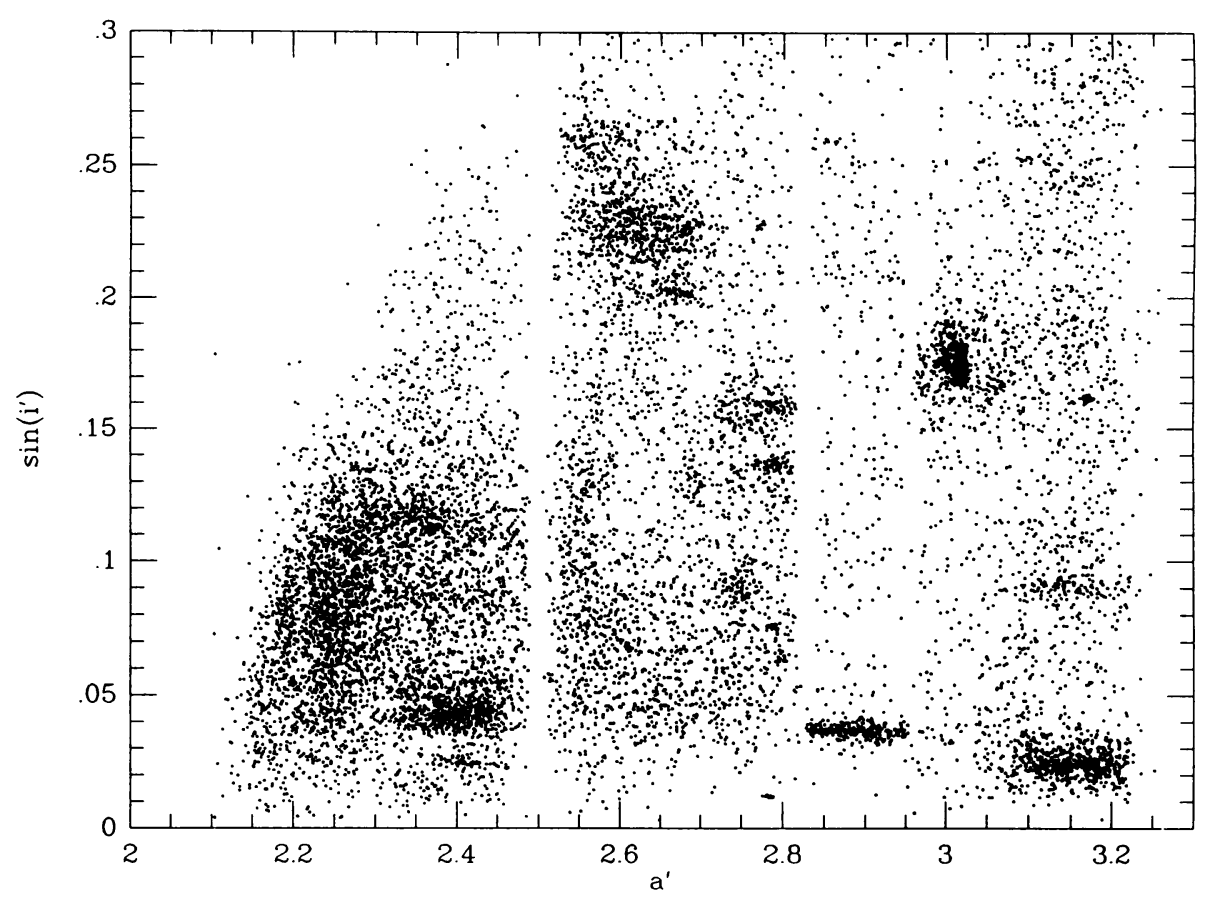

Fig. 1. $\sin i^{\prime}$ vs. $a^{\prime}$ plot for the very large data set of asteroid proper elements (over 12,000 objects) provided by Milani and Knežević (1994)

should be groupings of objects for which both the statistical definition, based on the analysis of proper elements, and the physical definition based on observations confirming a common genetic relationship, coincide. With this definition of families, only a few of them can be presently definitely accepted, mainly due to the paucity of observational data on the surface properties (color indexes, albedos, taxonomic types) for most of the asteroidal population.

On the other hand, it is not easy to get satisfactory "proofs" of the existence of families in a purely physical sense. Even not taking into account the present lack of observational data, which will be hopefully overcome in the next years, some intrinsic problems remain : first, the outcome of an impact depends on the overall specific impact energy, i.e., the ratio between the kinetic energy of the projectile and the mass of the target : when very energetic impacts occur, the fragments may be ejected at high velocities, and spread over very large regions of the proper elements space. In these cases, what is formed is not properly a family, since no observable group of objects remains in the space of proper elements after the 


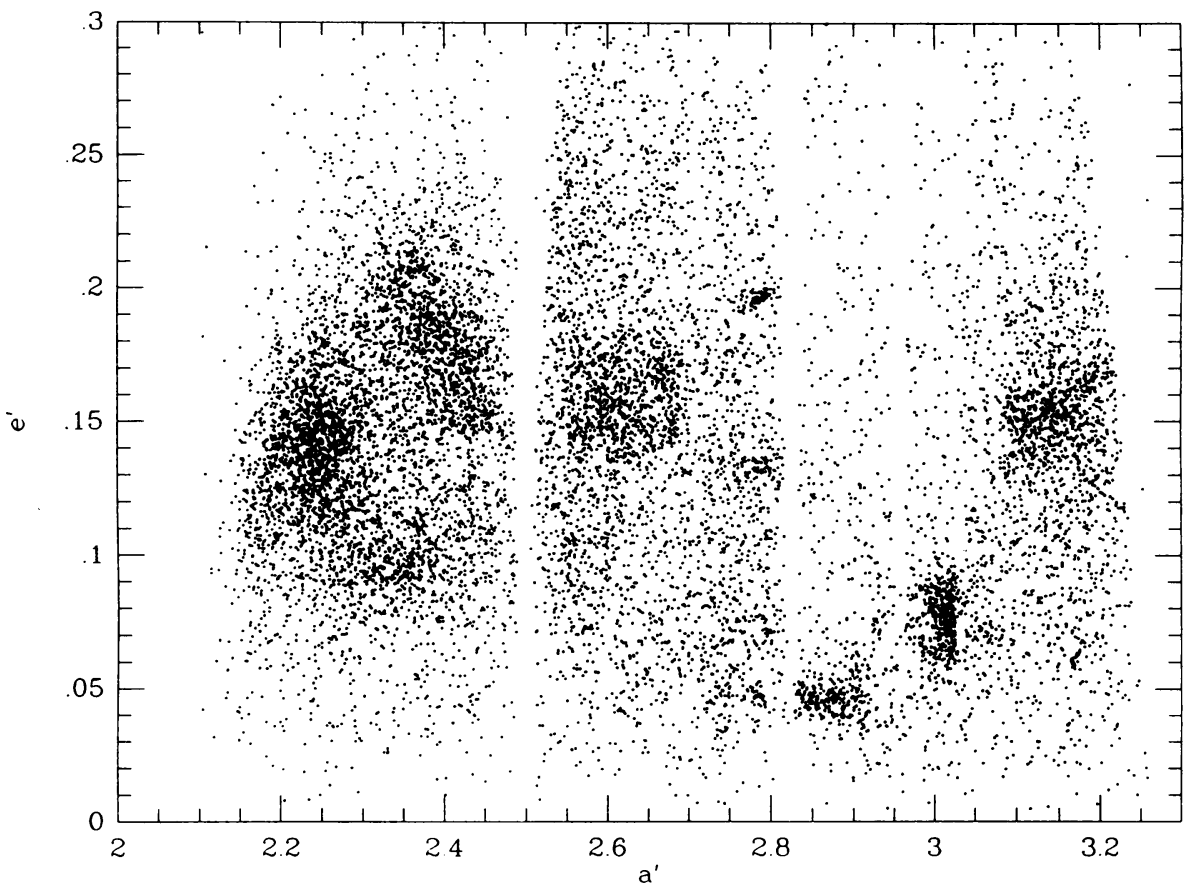

Fig. 2. $e^{\prime}$ vs. $a^{\prime}$ plot referring to the same data set of Fig. 2

impact. In some cases the particular mineralogical composition of the parent could lead to the formation of a number of fragments with well defined, and rare, surface properties, but these could not be identified as family members, in spite of their common collisional origin, due to their large spread. A few examples of this may be actually present in the asteroid belt (see, e.g., Burbine et al., 1992), and this subject has also deep implications for the general problem of the origin of meteorites. In other cases, however, just the opposite may occur : some well defined groupings of asteroids in the proper element space may be plunged in a background of objects bearing the same general surface properties. In this case, a physical confirmation may be more difficult. One should also take into account that some basic physical properties of collisional outcomes, like their mass and velocity distributions, are not presently well known due to our unsatisfactory knowledge of the physics of catastrophic impacts. On the other hand, some recent theoretical improvements related to the elaboration of complex hydrocode models (Melosh et al., 1992), or to the improvements of a semiempirical approach (Paolicchi et al., 1993), allow to be confident about the possibility to compare observational data with reliable 
theoretical predictions in the near future. Moreover, from the experimental point of view, the increasing amount of data obtained by means of sophisticated laboratory techniques (see Fujiwara et al., 1989 for a review) is encouraging. The next years will bring probably important results in the field of the physics of catastrophic collisions.

From the above discussion, it follows that the concept of family is based on concurring evidences coming from both statistics and physics : the first step in the process leading to the discovery of a family consists in identifying it as a statistically significant clustering of objects in the space of proper elements. The second step is to compare the physical properties of the supposed members with what is known about the outcomes of catastrophic impacts, and with the mineralogical properties of asteroidal bodies.

\section{Family identification : problems and recent improvements}

In the previous Section we have sketched a description of the ideal procedure which should be carried out for an unambiguous identification of asteroid families. In practice, however, the interplay of independent evidences coming from the statistical distribution of proper elements and from the observed physical properties of the supposed members has been rarely achievable so far.

This explains why for a long time, spanning over several tens of years, no definite agreement on the number of asteroid families, and on the memberships of these families, has been found by the authors who have published different family lists (see Valsecchi et al., 1989, for an exhaustive review). This does not mean that all these searches were wrong : actually, many of them led to interesting findings over many respects (we can quote the important work carried out by Williams, 1979, 1989 , as a good example of relevant contribution to the subject). The problem is that the results found by different searches contradicted each other.

Of course, this has entailed bad consequences for asteroidal science : as a matter of fact, a great deal of skepticism has arisen among many of those interested in the general process of collisional evolution of the asteroid belt, preventing them from seriously considering the constraints coming from the existence of the families. The skepticism was due both to the disagreement on the number of families identified by different authors (this number ranged from 15 to more than 100 on the basis of the papers published between 1978 and 1989) and to the observed cosmochemical inconsistency of some of the proposed families (Chapman et al., 1989).

On the other hand, some important improvements have been recently achieved from the dynamical and statistical point of view. For this reason, we have now a number of well established groupings of objects for which we are waiting for a definitive confirmation coming from observations. These groups, according to Farinella et al. (1992) cannot yet be called "families", and other terms such as "clans" or "clusters" appear more appropriate. On the other hand, on the basis of the first observational tests, the situation is encouraging, and some spectacular confirmations, like in the case of the Vesta family (Binzel and Xu, 1993), allow us to be very confident for the future developments.

All this has been made possible by the simultaneous development of independent statistical procedures of identification, and by the availability of new very 
large data bases of asteroid proper elements, computed by Milani and Knežević $(1990,1992)$ (versions from 4.2 to 5.7$)$.

These data have been exploited both by searches based on the D-criterion of Southworth and Hawkins (1963) (Lindblad, 1992), and by investigations based on new methods, introduced by Zappalà et al. (1990, 1994), and by Bendjoya et al. (1991), and Bendjoya (1993). These procedures are completely independent, and are based on the hierarchical clustering techniques of multivariate data analysis, and on the application of a mathematical technique called "wavelet analysis", respectively. We do not recall here the technical details of these procedures, which are extensively explained in the quoted papers. What we want to note, is that for the first time two independent techniques have been applied to the same (large) data base of asteroid proper elements, and have given results in good agreement with each other. Moreover, both procedures are mostly based on automatic algorithms managed by computer, and are easily reproducible and fairly "objective". In particular, their performances have been tested by means of computer simulations, in which fictitious families have been created and plunged in random backgrounds of different densities. The results have shown that both techniques behave well even in particularly difficult conditions, and are reliable in cases bearing a resemblance to the properties of the actual asteroid belt (Bendjoya et al., 1993).

These results are encouraging, taking into account that many searches for asteroid families carried out in the past by different authors, suffered from the (forced) adoption of small data bases on asteroid proper elements, and/or from the application of identification procedures mostly based on the (subjective) response of the human eye. Moreover, many of those searches did not include careful statistical methods for determining the reliability of the resulting families, or in some cases such criteria have been found to be questionable by other authors (such is the case of Williams' Poisson test, as will be noticed in the next Section).

Among other things, the most recent analyses have shown that, with large data sets of asteroid proper elements, some general, "philosophical" choices must be made, concerning the application of the identification methods. Apart from the obvious fact that groupings having no statistical significance have to be rejected, some problems can arise in some cases for the memberships of unambiguously identified groupings. In other words, the statistical criteria adopted can lead to different results due to the smooth transition that exists in the space of proper elements from the "core" of some families to the surrounding background. This implies that in several cases a statistically significant group is identified, but one has to make some choice about the criterion used to establish the identity of its members. One can be more conservative, and in this case only a fraction of the real members (the core of the cluster) will be accepted, but the resulting list will contain only a few chance interlopers, if any. The alternative is to be more liberal, and this implies to accept as family members also many objects lying in the outer part of the apparent cluster. In this case a larger number of "true" members will be recognized, but, as a drawback, one can expect also a significant amount of chance interlopers; the interlopers might account for a large fraction of the listed members in the worst cases (the "clans" according to the Farinella et al., 1992, terminology).

The problem of the uncertain membership of asteroid families cannot be solved 
by means of any general algorithm, since it reflects a real physical fact, that is the progressive mixing of the family members ejected with larger ejection velocities with the background of objects present in the surrounding zones of the belt. The best approach is probably the following : one has first to identify the reliable family candidates, with their "sure" members, i.e., those belonging to the cores of these groupings. The membership of the objects lying in the "halo" of the family could then be established by means of physical observations, allowing to exclude from the halo those objects which are not plausible on the basis of the observed characterization of the core objects. Of course, this approach cannot work when the family members cannot be discriminated, with respect to the background, on the basis of any observable property.

\section{The present situation}

There are currently several published lists of families. Among these, we have decided to focus our attention only on the most recent ones. On the basis of this choice, three main lists of asteroid families, obtained by means of different techniques, will be discussed in this Section. These lists are those given by Zappalà et al., 1994, Bendjoya, 1993, and Williams, 1989, 1992. Other independent searches have been recently performed by other authors, in particular Lindblad (1992), but the list of family memberships has not yet been published. The older family lists, published before 1989 (see Valsecchi et al., 1989) will not be analyzed here, even if we are aware that many of them have been historically important.

A comparison between the results obtained by Zappalà et al. (1994) by means of their most recent search based on the hierarchical clustering method, and by Bendjoya (1993), who used the most recent 3 -D version of his wavelet analysis approach, is encouraging. Actually, Zappalà et al., 1994, found 20 statistically significant and metric-independent family candidates, as well as 6 additional statistically significant candidates which were found to be metric-dependent, in the sense that their identification depends on the metric used to define the distance in the space of proper elements (see Zappalà et al., 1990, and Milani et al., 1992). In addition to these, 7 other groupings were found to be just below the threshold adopted for statistical reliability, and should be considered as dubious cases.

In the most recent application of his wavelet analysis method, Bendjoya (1993) found 26 statistically significant family candidates, plus 11 additional marginally significant groupings. The agreement between the resulting family lists can be considered good : taking into account just the groupings listed as statistically significant, those found in both the lists are 20; one of those listed by Zappalà et al. is listed as marginally significant in Bendjoya's list. Among the statistically significant groups listed by Bendjoya, 7 have no counterpart in the Zappalà et al. list. In most cases, however, they are small groupings; moreover, some of them are actually found by Zappalà et al. at the critical level of distance (Quasi Random Level QRL) adopted for the definition of reliable groupings, but they do not include the minimum required number of objects. The only one important discrepancy concerns a big group formed by 43 members, associated to the asteroid 79 Eurynome, found by Bendjoya, and which has no counterpart in the Zappalà et al. list. In three cases, 


\section{TABLE I}

Comparison between the family lists found by Bendjoya (1993) (B) and Zappalà et al. (1994) (Z). The columns give, respectively, the identification $\operatorname{Id}(B)$ and number of members $N_{B}$ of each grouping found by $\mathrm{B}$; the corresponding identification, $\operatorname{Id}(\mathrm{Z})$, and number of members $N_{Z}$ found by $\mathrm{Z}$; the percentage of the common members with respect to $N_{B}$ and $N_{Z}$; some remarks concerning a possible improvement of the agreement by relaxing the adopted criteria : for the criteria "2" and " 3 " of B see Bendjoya (1993); for the definition of the critical distance level QRL and its associated $\sigma(\mathrm{Z})$ see the text.

\begin{tabular}{|c|c|c|c|c|c|c|}
\hline $\operatorname{Id}(B)$ & $N_{B}$ & $\operatorname{Id}(Z)$ & $N_{Z}$ & $\%(\mathrm{~B})$ & $\%(Z)$ & Remarks \\
\hline 8 & 368 & $8+244^{* *}$ & 494 & 95 & 71 & crit.2 (B) : $95 \%(B), 85 \%(Z)$ \\
\hline 4 & 79 & 4 & 64 & 71 & 88 & \\
\hline 163 & 12 & 163 & 11 & 92 & 100 & \\
\hline 272 & 21 & $1726+363$ & 18 & 86 & 100 & \\
\hline 170 & 52 & 170 & 50 & 90 & 94 & \\
\hline 668 & 44 & 668 & 41 & 93 & 100 & \\
\hline 145 & 26 & 145 & 28 & 100 & 93 & \\
\hline 1272 & 37 & 1272 & 32 & 84 & 97 & \\
\hline 15 & 156 & 15 & 180 & 96 & 83 & crit.2 (B) : $95 \%(\mathrm{~B}), 93 \%(\mathrm{Z})$ \\
\hline 410 & 11 & 1403 & 8 & 73 & 100 & $Q R L+1 \sigma(\mathrm{Z}): 82 \%(\mathrm{~B}), 100 \%(\mathrm{Z})$ \\
\hline 490 & 12 & 490 & 11 & 92 & 100 & \\
\hline 221 & 219 & 221 & 273 & 100 & 80 & crit.2 (B) : $99 \%(B), 93 \%(Z)$ \\
\hline 158 & 161 & 158 & 207 & 100 & 78 & crit.2 (B) : $100 \%(B), 90 \%(\mathrm{Z})$ \\
\hline 135 & 145 & 44 & 221 & 100 & 66 & crit.2 (B) : $96 \%(B), 81 \%(Z)$ \\
\hline 213 & 17 & 808 & 10 & 59 & 100 & $Q R L+1 \sigma(\mathrm{Z}): 71 \%(\mathrm{~B}), 100 \%(\mathrm{Z})$ \\
\hline $1644^{*}$ & 11 & 1644 & 6 & 45 & 83 & $Q R L+1 \sigma(\mathrm{Z}): 82 \%(\mathrm{~B}), 90 \%(\mathrm{Z})$ \\
\hline 24 & 177 & 24 & 387 & 100 & 46 & $\begin{array}{l}\text { crit.2 (B) : } 100 \%(B), 71 \%(\mathrm{Z}) \\
\text { crit.3 (B) : } 98 \%(B), 83 \%(\mathrm{Z})\end{array}$ \\
\hline 10 & 29 & 10 & 20 & 66 & 95 & $Q R L+2 \sigma(\mathrm{Z}): 90 \%(\mathrm{~B}), 72 \%(\mathrm{Z})$ \\
\hline 569 & 13 & $569^{* *}$ & 6 & 46 & 100 & $Q R L+2 \sigma(\mathrm{Z}): 62 \%(\mathrm{~B}), 100 \%(\mathrm{Z})$ \\
\hline 847 & 35 & $847+2354$ & 16 & 40 & 88 & $Q R L+2 \sigma(\mathrm{Z}): 66 \%(\mathrm{~B}), 82 \%(\mathrm{Z})$ \\
\hline 79 & 43 & - & - & & & in 44 at $Q R L+3 \sigma(\mathrm{Z})$ \\
\hline 9 & 11 & - & - & & & 5 obj. at $Q R L+1 \sigma(\mathrm{Z})$ \\
\hline 27 & 17 & - & - & & & 6 obj. at $Q R L+2 \sigma(\mathrm{Z})$ \\
\hline 779 & 8 & - & - & & & 8 obj. at $Q R L+2 \sigma(\mathrm{Z})$ \\
\hline 1639 & 5 & - & - & & & 4 obj. at $Q R L(\mathrm{Z})$ \\
\hline 262 & 21 & - & - & & & $6 \mathrm{obj}$. at $Q R L(\mathrm{Z})$ \\
\hline 869 & 9 & - & - & & & $3 \mathrm{obj} . Q R L(\mathrm{Z})$ \\
\hline 137 & 19 & 137 & 10 & 53 & 100 & $\begin{array}{l}\text { tribe for B } \\
\text { crit.2 (B) : } 63 \%(B), 100 \%(\mathrm{Z})\end{array}$ \\
\hline
\end{tabular}

* : possible subgroup of 262 (B)

** : statistically reliable grouping with one metric only (see text) 
finally, one single group listed by Bendjoya includes two groups listed separately by Zappalà et al. As for the membership, the agreement is satisfactory, although, as explained in the previous Section, each statistically significant group should be separately studied in detail, beyond the "official" lists of members which constitute the output of the algorithms; actually, each candidate family has its own unique features, thus its structure should be analyzed separately. Even taking into account these caveats, however, the numbers and identities of family members published in the two searches are in a good agreement.

In Table I, the family candidates found by the two classifications are divided into three main categories : the first includes groupings for which the percentage of common members exceeds $70 \%$ of the number of members found by both classifications. The second category includes groupings for which the percentage of common members does not reach $70 \%$ of the number of members found by one of the two methods. In several cases, a better agreement is reached by relaxing slightly the criteria adopted for the definition of the membership, e.g. by using the so-called "criterion 2" (or "criterion 3") of Bendjoya, or accepting members above the nominal QRL of Zappalà et al. The third category includes the candidate families which are identified by one classification, but are not statistically reliable for the other (although they may be still recognizable, by relaxing the reliability criteria).

On the basis of these results, it appears that 20 good family candidates have been found in the asteroid belt. In addition to the three well known, "historical" families of Eos, Themis and Koronis, already found in the pioneering work of Hirayama at the beginning of the century (see references in Valsecchi et al., 1989), a number of other interesting cases are apparent. Some of these were already found in the past by some of the authors who produced the older family lists, while others are new, like the interesting grouping associated to the large asteroid 10 Hygiea (see later).

Interestingly, these 20 family candidates include also, with one exception, the 14 main families listed by Lindblad (1992) in his recent analysis based on the D-criterion applied to the 4.2 version of the asteroid proper elements data base provided by Milani and Knežević. Actually, Lindblad (1992) did not publish a list of the members of his families; however, the names of the lowest-numbered members of each family, used to identify them, correspond to those found by Zappalà et al. and Bendjoya. The only exception is given by the group of Goberta, which was listed by Lindblad, but belongs probably to the Themis family found by the other authors. Due to the lack of additional information about Lindblad's results, it is not possible to perform here a more detailed comparison of the results. Lindblad (1992) noticed also that he found a relevant number of groupings formed by just a few members, but the statistical significance of such groupings is not clear.

Given the good agreement between the classifications of Bendjoya and Zappalà et al. (agreement that probably can also be extended to Lindblad's classification), a few comments should be made about the disagreement existing with the results of the extensive searches for asteroid families carried out over more than ten years by Williams $(1979,1989,1992)$. These papers should be considered as milestones in the history of family searches, since Williams was able to compute accurate proper elements for a large data set of asteroids, and used this list for his comprehensive and systematic search work. He found a very high number of families, about 100 , 
many of which are composed by a few members. Such a result has been criticized by other authors (including ourselves) who did not find some of these families plausible, due to the presence of assemblages of different taxonomic types not compatible with a common collisional origin. The high number of families found by Williams is in disagreement with the results of most of the other searches carried out recently, including, of course, Zappalà et al. (1994) and Bendjoya (1993).

The reasons for such a disagreement should be analyzed and understood correctly, in order to avoid possible misunderstandings and consequent criticism about the overall meaning and reliability of the existing family classifications.

The representation of families in terms of the "stalactite diagrams" introduced by Zappalà et al. $(1990,1994)$ appears here particularly useful. This kind of diagrams show the sizes of the groupings existing in a given region of the space of proper elements (corresponding to different zones of the asteroid belt) as a function of the level of the mutual distance $D$ between the objects. The distance $D$ is used for the identification of the groupings in the framework of the hierarchical clustering method; we recall here that in these diagrams, due to the relation between the components of the ejection velocities of fragments and the resulting final differences in the proper elements, the distance is expressed in $\mathrm{m} / \mathrm{s}$ (see Zappalà et $a l ., 1990$, and Milani et al., 1992). From these diagrams, it is easy to see what kind of changes in the structure of a given grouping take place at different levels of $D$. A typical example is given in Fig. 3, which shows the different behavior exhibited by a typical "cluster" (very compact and stable at very different values of $D$ ) and a typical "clan", according to the nomenclature suggested by Farinella et al. (1992). The membership of the candidate families, identified by the hierarchical clustering method, is given by the objects belonging to the groupings found at a particular level of $D$, the so-called Quasi-Random Level (QRL). The QRL is determined on the basis of the stalactites which can be obtained in the different zones of the proper elements space by populations of fictitious objects distributed at random (on the scale of typical family sizes), but satisfying the constraint of reproducing the large-scale distributions of proper elements of the real asteroids.

The agreement (or disagreement) between different classifications can be investigated by analyzing the structure of the resulting groupings at different values of $D$, in order to find out whether the discrepancies between classifications obtained by means of different techniques may be due to the different criteria used for the acceptance/rejection of candidate families. In other words, we can check whether the discrepancies may be due to the fact that different authors "cut" a given stalactite at different levels.

We recall that in the case of the differences between the Zappalà et al. (1990) results and those obtained by Williams $(1989,1992)$ the disagreement cannot be due to the different sets of proper elements, as shown by Zappalà et al. (1992): the problem must be due to the different statistical methods used for family identification.

While the methods of hierarchical clustering and of wavelet analysis are both based on automated procedures managed by computer, the first step of the method of Williams was a visual examination of stereo plots, leading to the identification of family candidates. A statistical test was performed on the chosen clusters only 


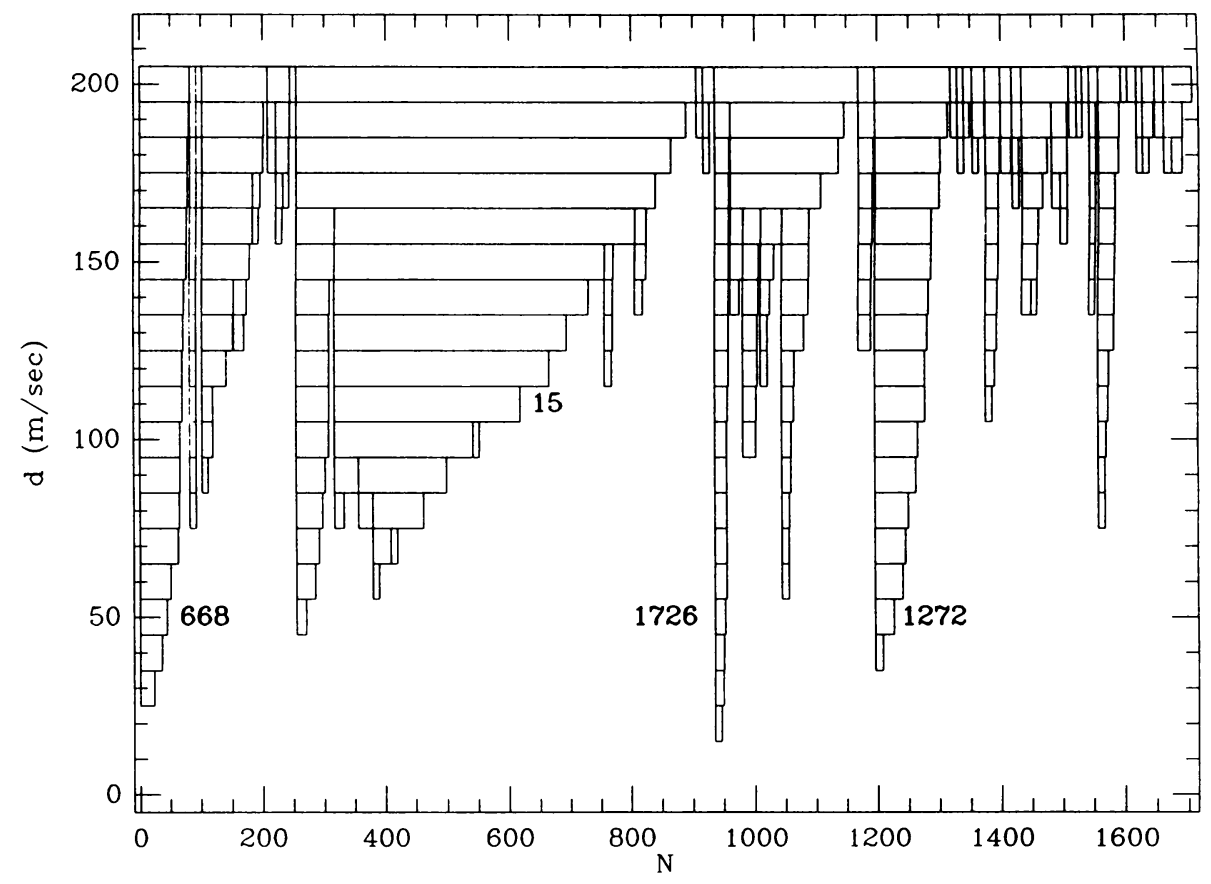

Fig. 3. A typical stalactite diagram, referring to the asteroids of the data set provided by Milani and Knežević (1994), in the zone of the belt delimited by $a^{\prime}=2.5$ and $a^{\prime}=2.825$. The size of the groupings found at each level of distance (expressed in $\mathrm{m} / \mathrm{s}$, see text) is shown in the abscissa. The critical Quasi Random Level (QRL) has not yet been determined for this data set, but should be around $140-150 \mathrm{~m} / \mathrm{sec}$. The differences between the behavior of a typical clan, the one associated to the asteroid 15 Eunomia, and some typical clusters, like those associated to the asteroids 668 Dora, 1726 Hoffmeister and 1272 Gefion, are evident (see the text)

a posteriori. He used Poisson statistics, based on the number of objects belonging to a cluster and the number expected by chance, derived from the background density and cluster volume. To be accepted as a family, the logarithm of the Poisson probability associated to a given identified clustering was required to be less than -3.5. 102 clusters passed this significance test.

It is our opinion that such a procedure is not completely safe, since Poisson statistics of density fluctuations would require a medium homogeneous on a large scale (and this is not the case in the present situation); moreover, the "boxes", in which a comparison is made between the number of objects really present and that expected on the basis of the average density, should have fixed sizes decided $a$ 
priori. Choosing the boxes a posteriori on the basis of the volume of each observed surdensity, leads to maximize the derived probability of the groupings to be not due to chance.

However, we do not want here to discuss in detail the efficiency and/or the validity of this method, but only investigate the possible sources of the discrepancies between the results obtained in this way and those coming from the other techniques of family identification; in particular, we will refer to the hierarchical clustering method, since for this we have a direct experience of its mechanisms and we can more easily compare the results by using the stalactite diagrams.

To do this, we introduce a "quality code" $q$, describing the agreement - family by family - of the Williams (W) and Zappalà et al. (Z) classifications at different significance levels in the $\mathrm{Z}$ stalactite representation. If $N_{i n t}$ is the number of members found to belong to a given family in both classifications (i.e., the intersection of a common $\mathrm{Z}$ and $\mathrm{W}$ family) and $N_{w i l}$ is the number of numbered objects found in the W family (we recall that Williams considered also a number of PLS objects which are not present in the data base used by Zappalà et al.) we assume to have a reasonable agreement when $N_{i n t} / N_{w i l}$ is larger than or equal to $1 / 3$. Families behaving in this way at the nominal QRL of $\mathrm{Z}$ have been defined of quality $q=$ 1. Since the QRL values were found by random simulations and are subject to a statistical variance $\sigma$, to families showing a satisfactory agreement $\left(N_{\text {int }} / N_{w i l} \geq\right.$ $1 / 3$ ) within $2 \sigma$ from QRL (i.e. cutting the $\mathrm{Z}$ stalactite at a more liberal significance level) we assigned a quality code $q=2$, while $q=3$ was assigned to families not reaching a satisfactory agreement within 3- $\sigma$ from QRL. In other words, quality codes 2 and 3 mean that in order to find a satisfactory agreement between the $Z$ and $\mathrm{W}$ classifications, we should accept in the framework of the $\mathrm{Z}$ method also groupings of very poor or no statistical significance.

On the other hand, we assume as a significance parameter for the $\mathrm{W}$ classification the logarithm of the Poisson probability $(\log \mathrm{P})$ obtained by the $\mathrm{W}$ statistical test. Then, we divide the $\mathrm{W}$ families in three categories : the high significance families having $\log \mathrm{P}<-10$; the intermediate ones, having $-10<\log \mathrm{P}<-5$; and the low significance families, having $\log \mathrm{P}>-5$. At this point, we can make a comparison between these two sets of parameters. In particular, for each of the three categories of $\mathrm{W}$ families we check the number of families having quality codes 1,2 or 3 (see Fig. 4). Note that the first category contains 27 families, the second 38 and the third 33 (for a few families of $W$ we could not compute the $q$ parameter, since they refer to high inclination asteroids, not present in the data set investigated by $Z$ ).

In general, we see that there is a good correlation between $q$ and $\log P$, in the sense that when $\log P$ increases the quality tends to be poor and viceversa. In particular, for $\log \mathrm{P}>-5$ (smaller significance in the W Poisson test) we have $85 \%$ (23) of the families with $q=2$ or 3 (poor or no intersection between the $Z$ and $W$ classifications), while for $\log \mathrm{P}<-10,79 \%$ of the families (26) have quality code $q=1$. Very interesting is the fact that these 26 families overlap almost perfectly to the set of high-reliability families found by Zappalà et al. and Bendjoya.

How to interpret this result in terms of the reliability of the various classifications? We have two possibilities : either to consider too optimistic the value of -3.5 (actually used by Williams) as a significance limit for $\log \mathrm{P}$ in the $\mathrm{W}$ classification 


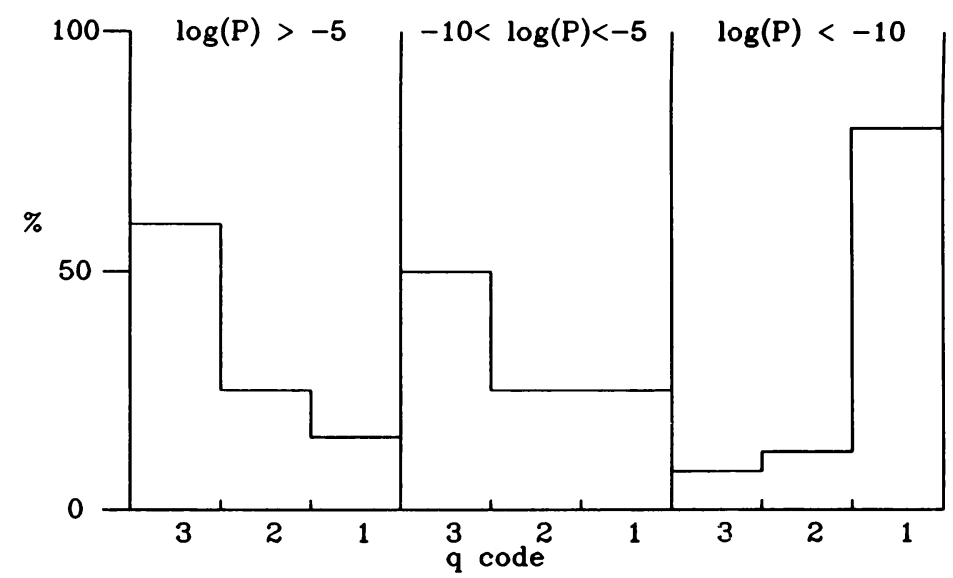

Fig. 4. A comparison between the results of the family classifications carried out by Williams $(1989,1992)$ and by Zappalà et al. (1994) shows that the agreement is very good ( $85 \%$, corresponding to 26 family candidates with $q=1)$ for the groupings having the smallest values of $\log (P)$ listed by Williams. The agreement is not as good (majority of groupings having $q=2$ or $q=3$ ) for higher values of $\log (P)$ (see text).

(or also to consider not completely correct the a posteriori use of a Poisson test to groupings already selected by visual inspection); or to consider too pessimistic the nominal significance level (QRL) used by Zappalà et al. (1994). Obviously, as coauthors of the latter paper, we tend to be in favor of our own choice, which we recall - has been tested by means of extensive numerical simulations (Bendjoya et al., 1993). However, we do not want, after having tried to be as "objective" as possible with the identification procedure, to be now biased in excluding all the Williams families which do not agree with our results ! What is really important to stress is that the frustrating discrepancy between different classifications, which in some way hindered the research on families for several years in the past, can be interpreted now satisfactorily in most cases. In general, the present situation is characterized by a satisfactory agreement between the results obtained by means of the application of identification algorithms managed by computer. The remaining discrepancies concern mostly the statistical reliability of a significant number of groupings identified visually, due to differences in the optimistic/pessimistic criteria used to interpret the results.

Therefore, what we suggest is the following : to consider at present as highly reliable only the families found by the Zappalà et al. (1994) and by the Bendjoya (1993) classifications (which are also essentially coincident with the most significant candidates identified by Lindblad, 1992). The majority of the Williams' families should be put in a "waiting list" : we cannot exclude at all that some (if not most) of the smaller Williams' families could be real, even if they have not been found by other searches. But we think that at present their existence cannot be claimed on the basis of a solid statistical evidence. A strong increase in the number of asteroids having high-quality proper elements, in particular the addition of many smaller 
objects which have been so far excluded from the analyses, will certainly permit to discriminate the "good" candidates among the presently existing controversial cases.

\section{Open problems and conclusions}

The most recent family searches have indicated that two main types of plausible candidate families are apparent : the clusters have sharp limits, and their membership is particularly reliable, as it does not depend significantly on the criterion used to discriminate between family members and background objects in the space of proper elements. The clans are large associations, having very uncertain boundaries, and often characterized by the presence of several subgroupings. Their memberships cannot be defined easily without an adequate observational support. Five clans have been reliably identified so far; they are named after their lowest-numbered member : Flora, Nysa, Eunomia, Hygiea and Vesta. Clans are a challenging subject for future studies. It would be interesting to understand whether their structures are the consequences of some peculiar collisional events, or if they were generated by multicollisional phenomena. The observational data so far available do not allow to draw any definite conclusion, as remarked by Cellino and Zappalà (1993). Of course, in the cases of Vesta and Hygiea, due to the large sizes of their parent bodies $(400-500 \mathrm{~km}$ diameter $)$, the origin of the families is probably related to energetic, almost catastrophic, cratering events. It is hard to state whether the impact responsible for the formation of the Vesta family, which is also probably related to the hemispheric-scale albedo variegation detected by the observations (Cellino et al., 1987; Cellino et al., 1989), has been energetic enough to fracture the interior of this asteroid. Practically no data are available in the case of the Hygiea clan, located in the outer part of the main belt. Here, an observational effort should be carried out, since there are several analogies with the Vesta event.

The case of the Flora clan $\left(a^{\prime} \sim 2.25 \mathrm{AU}\right)$ remains puzzling. Figures 1 and 2 show that this clan is located in a very crowded region of the inner belt. The accuracy of the proper elements in this zone is a little worse with respect to the other regions of the main belt, due to the presence of several resonances. For this reason, a reliable identification of this clan, and an assessment of its main features, is not easy. With a smaller data set of proper elements, with respect to the one which is presently available, and using also a less evolved version of the hierarchical clustering method, Zappalà et al. (1990) were not able to unambiguously identify this clan as a single statistically reliable grouping. On the other hand, an analysis of the asteroid size distribution in the main belt performed by Cellino et al. (1991) showed that the size distribution within the Flora region is very similar to that characterizing the major families (Eos, Themis and Koronis). Finally, Zappalà et al. (1994), as well as Bendjoya (1993) list now unambiguously the Flora group as one of the main clans present in the asteroid belt.

Another interesting case is the one of the Nysa clan. While Nysa itself (an E-type object) should be considered as a probable interloper, as well as the other large $M$ asteroid Hertha, this clan is characterized by the presence of a large fraction of the presently known asteroids belonging to the rare $\mathrm{F}$ taxonomic type (see also Cellino 


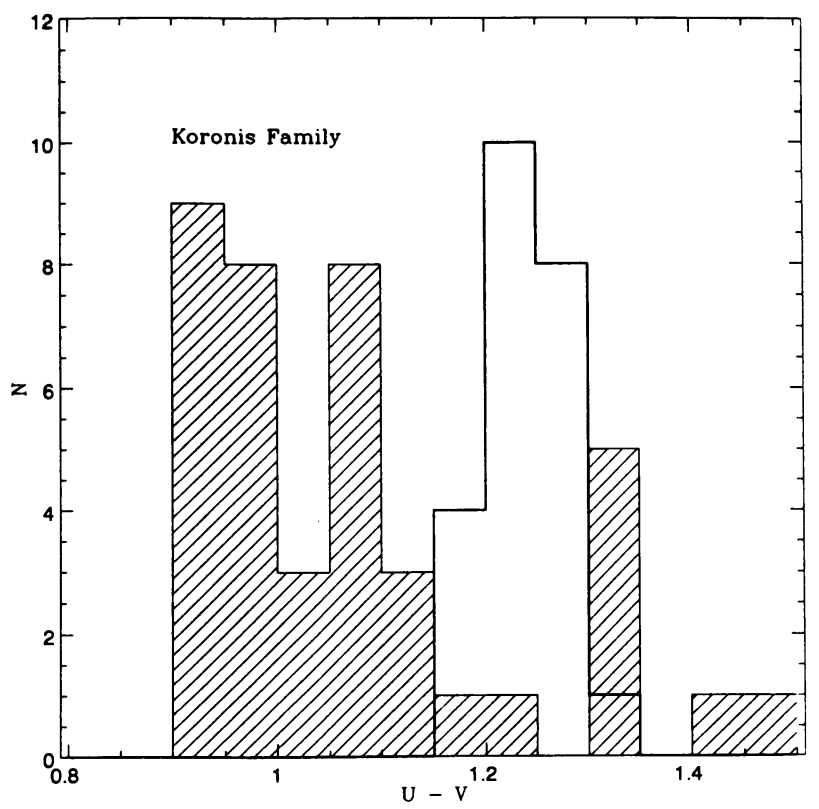

Fig. 5. U - V distribution for the members of the Koronis family (empty histogram), and for the background objects of the same zone of the belt (shaded histogram). The family appears well separated from the background.

and Zappalà, 1993). This seems very interesting, also taking into account that some F-type asteroids are present among the population of Near-Earth-Asteroids and that the clan lies close to the inner edge of the $3: 1$ resonance. Here, a specific observational campaign appears particularly useful and promising.

Figures 5-7 show some other facts which deserve further investigations. It is easy to see that both the Koronis and the Eos families show some peculiar distributions of the color-index $\mathrm{U}-\mathrm{V}$ and the albedo $p_{V}$, with respect to the background objects of the zones in which they are located. The U-V distributions of these families are noticeably similar, being peaked at values of about 1.25 . The Koronis members for which an evaluation of the taxonomic type is known, are classified as $S$, but they could constitute a definite subset of the $S$ objects. As for the Eos family members, the available data indicate that they belong to the rare $\mathrm{K}$ taxonomic type. In addition to the U-V peculiarities, the Eos members have also albedo values well distinct with respect to the background objects in their zone of the belt (Fig. 7). This observational evidence should be kept in mind, since probably it is not due to chance, but reflects some important facts about the mineralogical differentiation of asteroid interiors, as well as, possibly, on the influence of collisional events on the observable optical properties of the asteroid surfaces.

Of course, the well established family candidates constitute a challenge for the 


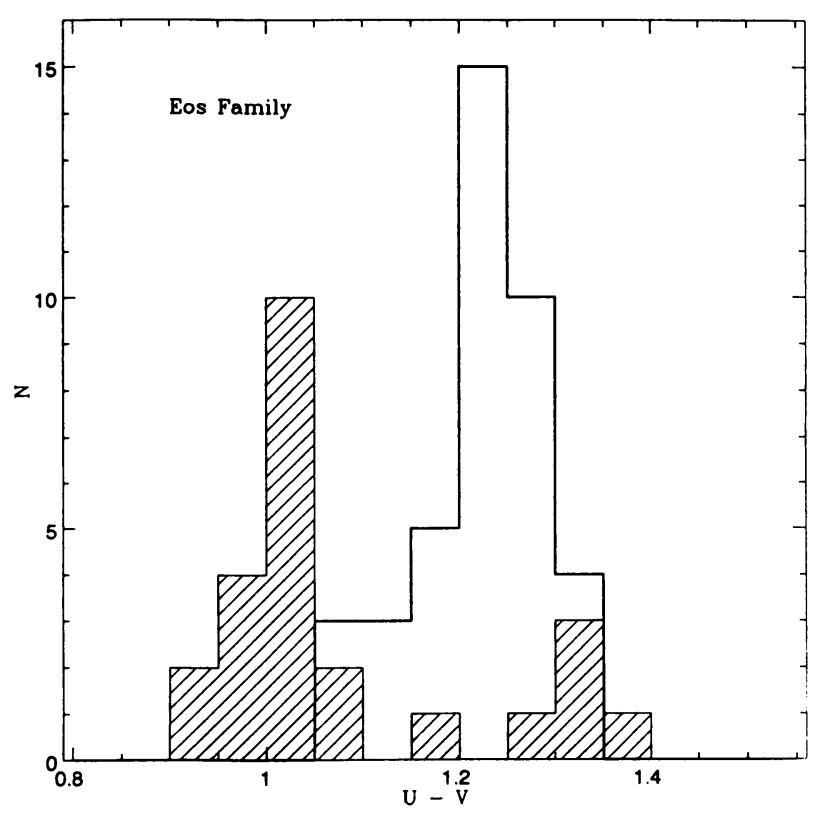

Fig. 6. The same as Fig. 5, but for the family of 221 Eos.

modern asteroidal science. On one hand, they deserve some dedicated observational campaigns, in order to derive the necessary information about the optical properties of the surfaces of the supposed members. This in turn would allow to discriminate in many cases between the "real" members and the chance interlopers. On the other hand, the families can provide the main observational evidence about the outcomes of catastrophic disruptions of bodies for which self-gravitation is not negligible. This would be very useful, if we take into account that recently a big effort has been made in order to improve the present physical understanding of collisional events (Melosh et al., 1992, Asphaug and Melosh, 1993, Paolicchi et al., 1989, 1993, 1994). Potentially, this can entail a better comprehension of the overall scenario of the collisional history of the asteroid belt. In particular, the abundance and size distribution of the presently identifiable families put some important constraints on any general model of the collisional evolution (see Davis et al., 1989, for a review). Taking into account the case of the Vesta family, it is reasonable to expect that an analysis of the most important family candidates, especially in the case of the clans, could give some hints concerning the origin of the Near-Earth-Asteroids. In this respect, as stated above, the Nysa clan, with its large amount of F objects, looks particularly promising.

Of course, a number of problems have to be faced and should be solved : an example is given by the asymmetries in the ejection velocities of fragments from the catastrophic breakups which led to the formation of the present families. This 


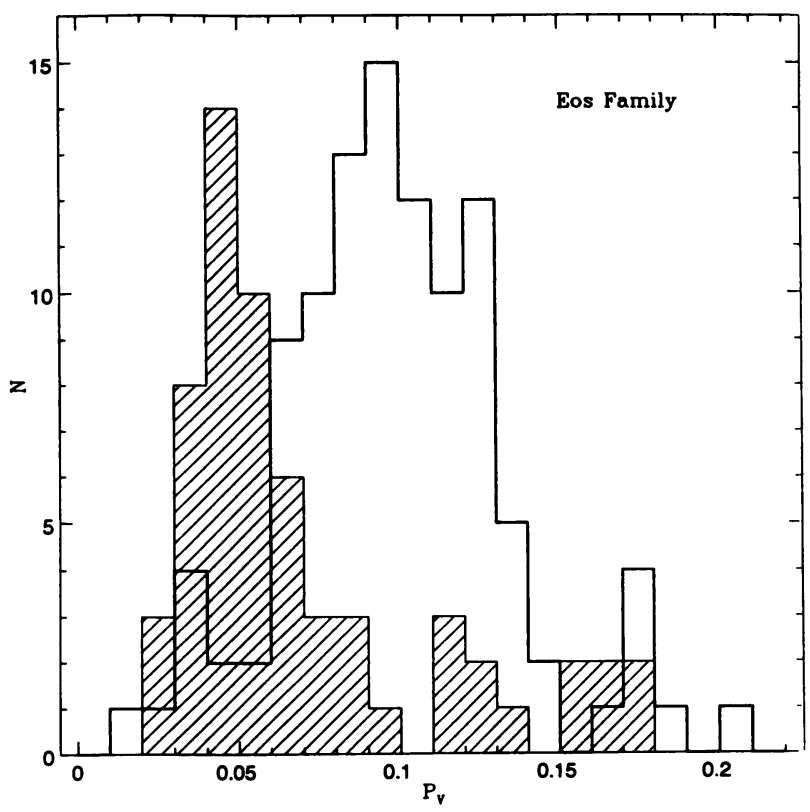

Fig. 7. Albedo $p_{V}$ distribution for the members of the Eos family (empty histogram) and for the background objects in the same zone of the belt (shaded histogram).

problem has been known for many years (Brouwer 1951, Zappalà et al., 1984, Chapman et al., 1989). An analysis of the ejection velocity distributions resulting from the new, reliable candidate families seems now necessary.

Finally, we note that the continuous refinement of the techniques of asteroid proper elements computation, which has triggered the recent improvement of the situation concerning the identification of asteroid families, is not over. It is now possible to carry out a new extensive family search, based upon a data set of over 12,500 objects (Milani and Knežević, 1994; Knežević and Milani, this volume). Of course, this will be done in the near future. Such a job should give some answers about the structures of the candidate families identified so far, and about the possible statistical significance of some groupings which are presently marginal. Even the objects at high orbital inclination should now be accessible (Lemaitre and Morbidelli, 1994).

We can conclude this review by saying that the epoch of skepticism about asteroid families seems really over. We expect the near future to be very exciting, with large amounts of new data which will improve our understanding of the history of the asteroidal population, and its interrelationships with the other minor bodies of the solar system. 


\section{References}

Asphaug, E. and Melosh, H.J. : 1993, "The Stickney impact of Phobos : a dynamical model." Icarus, 101, 144-167.

Bendjoya, Ph. : 1993, "A classification of 6479 asteroids into families by means of the wavelet clustering method." Astron. Astrophys. Suppl. Series, 102, 25-55.

Bendjoya, Ph., Slezak, E. and Froeschlé, Cl. : 1991, "The wavelet transform : a new tool for asteroid family determination." Astron. Astrophys., 251, 312-330.

Bendjoya, Ph., Cellino, A., Froeschlé, Cl. and Zappalà, V. : 1993, "Asteroid dynamical families : a reliability test for two identification methods." Astron. Astrophys, 272, 651-670.

Binzel, R.P. and Xu, S. : 1993, "Chips off of asteroid 4 Vesta : evidence for the parent body of basaltic achondrite meteorites." Science, 260, 186-190.

Broglia, P. and Manara, A. : 1989, "Rotational variations in the optical polarization of (4) Vesta." Astron. Astrophys., 214, 389-390.

Brouwer, D. : 1951, "Secular variations of the orbital elements of minor planets." Astron. J., 56, 9-32.

Burbine, T.H., Gaffey, M.J. and Bell, J.F. : 1992, "S-asteroids 387 Aquitania and 980 Anacostia : possible fragments of the breakup of a spinel-bearing parent body with CO3/CV3 affinities." Meteoritics, 27, 424-434.

Cellino, A. and Zappalà, V. : 1993, "Asteroid clans : super families or multiple events ?", Celest. Mech., 57, 37-47.

Cellino, A., Zappalà, V., Di Martino, M., Farinella, P. and Paolicchi, P. : 1987, "Flattening, pole and albedo features of 4 Vesta from photometric data." Icarus, 70, 546-565.

Cellino, A., Di Martino, M., Drummond, J., Farinella, P., Paolicchi, P. and Zappalà, V. : 1989, "Vesta's shape, density and albedo features." Astron. Astrophys., 219, 320-321.

Cellino, A., Zappalà, V. and Farinella, P. : 1991, "The size distribution of asteroids from IRAS data." Mon. Not. Roy. Astr. Soc., 253, 561-574.

Chapman, C.R., Paolicchi, P., Zappalà, V., Binzel, R.P. and Bell, J.F. : 1989, "Asteroid families : physical properties and evolution." In Asteroids II, (R.P. Binzel, T. Gehrels, M. S. Matthews, Eds.), 386-415, Univ. of Arizona Press.

Cruikshank, D.P., Tholen, D.J., Hartmann, W.K., Bell, J.F. and Brown, R.H. : 1991, "Three basaltic Earth-approaching asteroids and the source of the basaltic meteorites." Icarus, 89, 1-13.

Davis, D.R., Weidenschilling, S.J., Farinella, P., Paolicchi, P. and Binzel, R.P. : 1989, "Asteroid collisional history : effects on sizes and spins." In Asteroids II, (R.P. Binzel, T. Gehrels, M. S. Matthews, Eds.), 805-826, University of Arizona Press.

Farinella, P., Davis, D.R., Cellino, A. and Zappalà, V. : 1992, "From clusters to families : a proposal for a new nomenclature." In Asteroids Comets meteors 1991, (A.W. Harris, E. Bowell, Eds.), 165-166, L.P.I., Houston.

Fujiwara, A., Cerroni, P., Davis, D.R., Ryan, E., Di Martino, M., Holsapple, K. and Housen, K. : 1989, "Experiments and scaling laws on catastrophic collisions." In Asteroids II, (R.P. Binzel, T. Gehrels, M. S. Matthews, Eds.), 240-265, University of Arizona Press.

Greenberg, R. and Nolan, M.C. : 1989, "Delivery of asteroids and meteorites to the inner solar system." In Asteroids II, (R.P. Binzel, T. Gehrels, M. S. Matthews, Eds.), 778804, University of Arizona Press.

Lemaitre, A. and Morbidelli, A. : 1994, "Calculation of proper elements for high inclined asteroidal orbits." Celest. Mech., in press.

Lindblad, B.A. : 1992, "A computer search for asteroid families." In Asteroids Comets meteors 1991, (A.W. Harris, E. Bowell, Eds.), 363-366, L.P.I., Houston.

Melosh, H.J., Ryan, E.V. and Asphaug, E. : 1992, "Dynamic fragmentation in impacts : hydrocode simulation of laboratory impacts." J. Geophys. Res., 97, 735-759. 
Milani, A. and Knežević, Z. : 1990, "Secular perturbation theory and computation of asteroid proper elements." Celest. Mech., 49, 247-411.

Milani, A. and Knežević, Z. : 1992, "Asteroid proper elements and secular resonances." Icarus, 98, 211-232.

Milani, A. and Knežević, Z. : 1994, "Asteroid proper elements and the dynamical structure of the asteroid main belt." Icarus, in press.

Milani, A., Farinella, P. and Knežević, Z. : 1992, "On the search for asteroid families." In Interrelations between Physics and Dynamics for Minor Bodies in the Solar System, (Cl. Froeschlé, D. Benest, Eds.), 85-132, Editions Frontières, Gif-sur-Yvette, France.

Paolicchi, P., Cellino, A., Farinella, P. and Zappalà, V. : 1989, "A semiempirical model of catastrophic breakup processes." Icarus, 77, 187-212.

Paolicchi, P., Verlicchi, A. and Cellino, A. : 1993, "Catastrophic fragmentation and formation of families : preliminary results from a new numerical model." Celest. Mech., 57, 49-56.

Paolicchi, P., La Spina, A., Paolicchi, P. and Cellino, A. : 1994, "The interpretation of laboratory experiments in the framework of an improved semi-empirical model." Planet. Space Sci., in press.

Southworth, R. and Hawkins, G. : 1963, "Statistics of meteor streams." Smithson. Contrib. Astrophys., 7, 261-285.

Valsecchi, G., Carusi, A., Knežević, Z., Kresák, Ľ. and Williams, J.G. : 1989, "Identification of asteroid dynamical families." In Asteroids II, (R.P. Binzel, T. Gehrels, M. S. Matthews, Eds.), 368-385, University of Arizona Press.

Williams, J.G. : 1979, "Proper elements and family memberships of the asteroids." In Asteroids, (T. Gehrels, Ed.), 1040-1063, University of Arizona Press.

Williams, J.G. : 1989, "Asteroid family identifications and proper elements." In Asteroids II, (R.P. Binzel, T. Gehrels, M. S. Matthews, Eds.), 1034-1072, University of Arizona Press.

Williams, J.G. : 1992, "Asteroid families - an initial search." Icarus, 96, 251-280.

Zappalà, V., Farinella, P., Knežević, Z. and Paolicchi, P. : 1984, "Collisional origin of asteroid families : mass and velocity distributions." Icarus, 59, 261-285.

Zappalà, V., Cellino, A., Farinella, P. and Knežević, Z. : 1990, "Asteroid families. I. Identification by hierarchical clustering and reliability assessment." Astron. J., 100, 20302046.

Zappalà, V., Cellino, A. and Farinella, P. : 1992, "A comparison between families obtained from different proper elements." In Asteroids Comets meteors 1991, (A.W. Harris, E. Bowell, Eds.), 675-678, L.P.I., Houston.

Zappalà, V., Cellino, A., Farinella, P. and Milani, A. : 1994, "Asteroid families. II. Extension to unnumbered multiopposition asteroids." Astron. J. 107, 772-801. 